\title{
Investigation of the Inertial Characteristics of the Railway Track System
}

\author{
Aditi Kumawat ${ }^{10000-1111-2222-3333]}$, Prishati Raychowdhury ${ }^{1[111-2222-3333-4444]}$, Sarvesh \\ Chandra ${ }^{1[1111-2222-3333-4444]}$ \\ ${ }^{1}$ Department of Civil Engineering, Indian Institute of Technology Kanpur, Kanpur 208016, In- \\ dia, aditi.iitkmailegmail.com
}

\begin{abstract}
Elastic foundation models offer a computationally efficient way for the qualitative analysis of the railway track system. However, the inertial characteristics of the foundation are neglected while modelling the railway track system using those models. This paper investigates the effect of incorporating the mass of the foundation on the behavior of the elastic foundation models under the dynamic train loading. The railway track system is idealized as an infinite EulerBernoulli beam resting on a continuous two-layer system with top and bottom layer denoting the ballast and subgrade, respectively. The ballast layer is modelled using inertial elastic shear elements and the subgrade by inertial viscoelastic elements. A time-domain deflection analysis of the proposed model is carried out for the various ranges of train speeds. It is found that the incorporation of the inertial characteristics of the sub-structural system may lead to significant underestimation in the critical velocity values (by up to $85 \%$ ). Further, the deflection magnitudes and the critical velocity of the system is found to be highly sensitive to the stiffness of the substructure. Higher deflection and lower critical velocity values are observed in the case of soft subgrade as compared to those in the stiff subgrade. Finally, the incorporation of the shear parameter associated with the ballast significantly decreases the deflection magnitudes.
\end{abstract}

Keywords: Elastic foundation models, Foundation mass, Dynamic load, railway track, analytical model

\section{Introduction}

Performance of the railway track structure under the dynamic train loading is a safety concern and needs to be taken into account while modeling the railway track systems (Esveld 2001). The elastic foundation models offer a computationally efficient way for the qualitative analysis of the railway track system under the effects of moving train loads (Chen and Huang 2000; Chen et al. 2001; Mallik et al. 2006; Basu and Rao 2013; Froio et al. 2018). Among those models, one of the most simplistic and commonly used models is the beam resting on Winkler foundation, also termed as the 'one-parameter' model. Various studies have been carried out to study the response of the one-parameter model when subjected to the various type of loading conditions. Timoshenko (1926) was among the first to analyze the response of a typical beam resting on Winkler type 
elastic foundation and subjected to harmonic moving load. Later, Kenney (1954) studied the response of a beam on Winkler foundation subjected to a concentrated load moving at a constant velocity. However, it is a well known fact that the Winkler model, although easy to use, does not capture the shear characteristics, inherent damping, and inertia-related effects associated with the foundation system.

In order to represent the railway track system more realistically, many researchers (e.g., Sun 2001) have incorporated the damping characteristics associated with the ground in the Winkler model. To further generalize the model, in several studies the interaction between the viscoelastic spring elements is introduced in the form of the Pasternak shear elements. For instance, Kargarnovin and Younesian (2004), Younesian and Kargarnovin (2009), and Basu and Rao (2013) idealized the track as a infinite beam resting on a viscoelastic foundation model comprising of springs with shear interactions. However, in all of the above-mentioned Winkler based elastic foundation models the inertial effects related to the substructure are either absent or incorporated by increasing the mass per unit length of the rail beam (Chen and Huang 2000; Chen et al. 2001).

In this paper the effect of incorporating the inertia of the foundation on the behavior of the elastic foundation models under the dynamic train loading is investigated. The railway track system is idealized as an infinite Euler- Bernoulli beam resting on a continuous two-layer system with top and bottom layer denoting the ballast and subgrade, respectively. The ballast layer is modelled using inertial elastic shear elements and the subgrade by inertial viscoelastic elements. A time-domain deflection analysis of the proposed model is carried out for the various ranges of train speeds.

\section{Analysis}

The model used to idealize the railway formation is presented in Fig. 1. The rail beam is modeled as an infinite Euler-Bernoulli beam overlying the sub-structure. The substructure is idealized as a continuous two-layer system with top and bottom layer denoting the ballast and subgrade, respectively. The ballast layer is modelled using inertial elastic shear elements and the

$$
E_{R} I_{R} \frac{\partial^{4} w}{\partial x^{4}}+q(x, t)+\rho_{R} \frac{\partial^{2} w}{\partial t^{2}}+c_{R} \frac{\partial w}{\partial t}=p(x, t)
$$

subgrade by inertial elastic elements. Further, the rail beam is subjected to point load of magnitude $P_{\text {wheel }}$ moving with a constant velocity $v$. The differential equation of motion of the rail beam under the above-described idealizations is given by

where $w(x, t)$ is the transverse deflection of the beam (m), $x$ is the space coordinate measured along the length of the beam (m), $t$ denotes time (s), $E_{R}$ is Young's modulus of beam material $\left(\mathrm{N} / \mathrm{m}^{2}\right), I_{R}$ is the moment of inertia of the beam cross-section about 
its neutral axis $\left(\mathrm{m}^{4}\right), q(x, t)$ is the reaction from ballast on the beam (in N/m), $\rho_{R}$ is the mass per unit length of the beam $(\mathrm{kg} / \mathrm{m})$, and $c_{R}$ denotes the coefficient of viscous damping per unit beam length $\left(N-s / \mathrm{m}^{2}\right)$. It may be noted that in the considered model the overall damping associated with the ballast and subgrade is incorporated via $c_{R}$. This method of incorporating the sub-structural damping is widely used in various analytical studies (e.g. Esveld 2001; Sun 2001; Mallik et al. 2006; Basu and Rao 2013) wherein the viscous damping associated with the substructure is considered equivalent to the viscous damping of the beam.

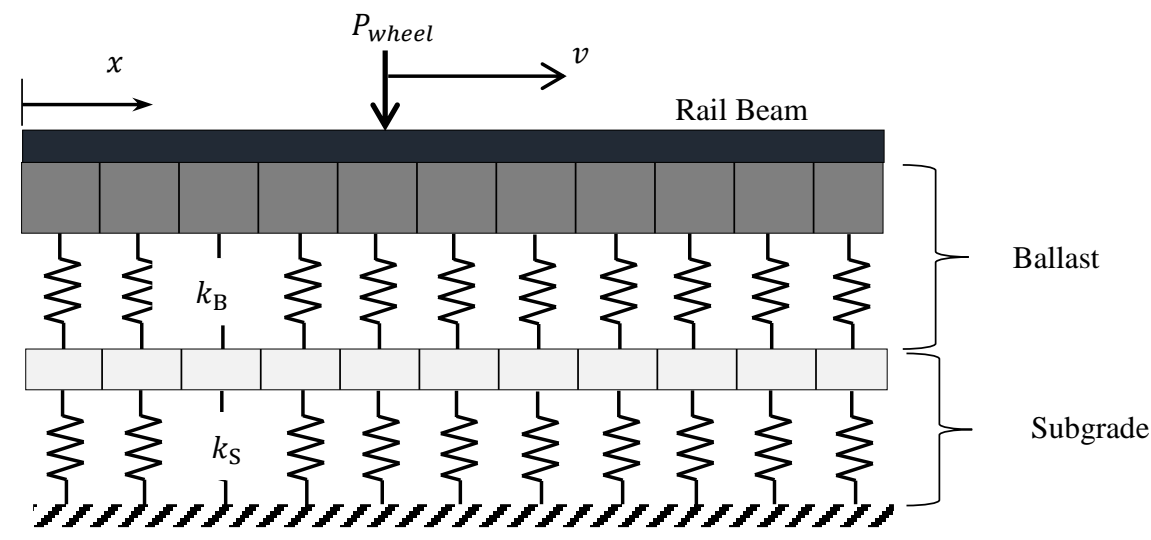

Fig. 1. Schematic diagram of the proposed model

Further, $p(x, t)$ denotes general time-varying distributed vertical load (N/m) acting on the sleeper beam and for the moving load case, $p(x, t)=P_{\text {wheel }} \delta(x-v t)$, where $\delta$ is the Dirac's delta function. Now, defining $f(\omega)$ as the Fourier transform of the function $f(t)$ such that

$$
\begin{gathered}
\hat{f}(\omega)=\int_{-\infty}^{\infty} f(t) e^{-\imath \omega t} d t \\
f(t)=\frac{1}{2 \pi} \int_{-\infty}^{\infty} \hat{f}(\omega) e^{\imath \omega t} d \omega
\end{gathered}
$$

where $\omega$ is the angular frequency and $\iota$ denotes $\sqrt{-1}$.

On taking Fourier transform of Eq. (1) using Eq. (2) and assuming that $w(x, t)$ and its time derivatives vanish at $t= \pm \infty$, the rail beam deflection equation is obtained in frequency domain as

$$
\begin{gathered}
E_{R} I_{R} \widehat{w}^{\prime \prime \prime \prime}(x, \omega)+\hat{q}(x, \omega)-\rho_{R} \omega^{2} \widehat{w}(x, \omega)+\iota c_{R} \omega \widehat{w}(x, \omega) \\
=\left(P_{\text {wheel }} / v\right) e^{-i \omega\left(\frac{x}{v}\right)}
\end{gathered}
$$

where $\widehat{w}(x, \omega)$ and $\hat{q}(x, \omega)$ denote the Fourier transforms of $w(x, t)$ and $q(x, t)$, respectively. 
The reaction from the ballast on the rail beam i.e., $q(x, t)$ depends on the mechanics of ballast and subgrade. Fig. 2(a) shows an infinitesimal element of the substruture at the location $x$. In this figure, $w(x, t)$ and $w_{1}(x, t)$ respectively denote the deflection of the rail beam/ballast layer element and subgrade. Let, $\left(\rho_{B}, k_{B}\right)$ and $\left(\rho_{S}, k_{S}\right)$ denote the mass per unit beam length and the stiffness of ballast and subgrade layers respectively.

Further, Fig. 2(b) shows the free body diagram of the ballast layer element. Here, $N_{x}$ denotes the shear force acting on the ballast layer element and is given by

$$
N_{x}=k_{1}(\partial w / \partial x)
$$

where $k_{1}$ is the shear parameter associated with the ballast layer $(N)$.

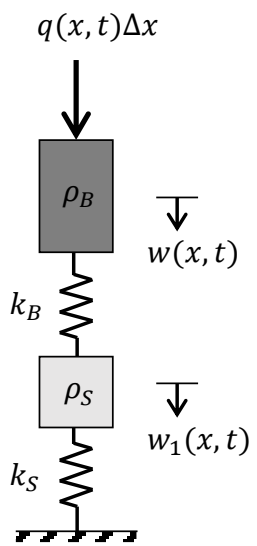

(a)

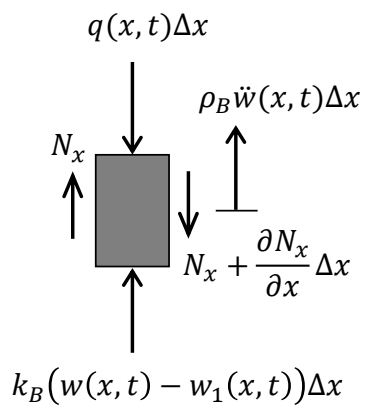

(b)

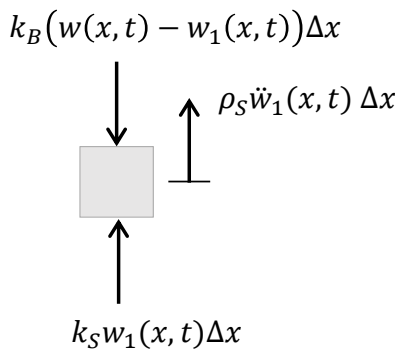

(c)

Fig. 2. Forces on the sub-structural elements

Using the above equation the vertical force equilibrium equation of the ballast element can be written as

$$
\rho_{B} \frac{\partial^{2} w}{\partial t^{2}}+k_{B}\left(w-w_{1}\right)-k_{1} \frac{\partial^{2} w}{\partial x^{2}}=q(x, t)
$$

Similarly, the free body diagram of the subgrade layer element is shown in Fig. 2(c). The vertical force equilibrium equation for this case is given by

$$
\rho_{S} \frac{\partial^{2} w_{1}}{\partial t^{2}}-k_{B}\left(w-w_{1}\right)+k_{S} w_{1}=0
$$

On taking the Fourier transform of Eqs. (6) and (7) using Eq. (2) we obtain

$$
\begin{aligned}
-\rho_{B} \omega^{2} \widehat{w}+k_{B}\left(\widehat{w}-\widehat{w}_{1}\right)-k_{1} \frac{\partial^{2} \widehat{w}}{\partial x^{2}} & =\hat{q} \\
-\rho_{S} \omega^{2} \widehat{w}_{1}-k_{B}\left(\widehat{w}-\widehat{w}_{1}\right)+k_{S} \widehat{w}_{1} & =0
\end{aligned}
$$

On solving Eqs. (7) and (8), the expression for $\hat{q}(x, \omega)$ is given by 


$$
\hat{q}=-k_{1} \frac{\partial^{2} \widehat{w}}{\partial x^{2}}+\left(-\rho_{B} \omega^{2}+\frac{k_{B}\left(-\rho_{S} \omega^{2}+k_{S}\right)}{\left(-\rho_{S} \omega^{2}+k_{B}+k_{S}\right)}\right) \widehat{w}
$$

Further, using the above equation we can define the frequency dependent equivalent modulus for the considered rail-track model as

$$
k(\omega)=\left(-\rho_{B} \omega^{2}+\frac{k_{B}\left(-\rho_{S} \omega^{2}+k_{S}\right)}{\left(-\rho_{S} \omega^{2}+k_{B}+k_{S}\right)}\right)
$$

Further, on substituting $\hat{q}(x, \omega)$ from Eq. (10) in Eq. (4) we obtain

$$
\begin{gathered}
E_{R} I_{R} \frac{\partial^{4} \widehat{w}}{\partial x^{4}}-k_{1} \frac{\partial^{2} \widehat{w}}{\partial x^{2}}+k(\omega) \widehat{w}-\rho_{R} \omega^{2} \widehat{w}(x, \omega)+\iota c_{R} \omega \widehat{w}(x, \omega) \\
=\left(P_{\text {wheel }} / v\right) e^{-i \omega\left(\frac{x}{v}\right)}
\end{gathered}
$$

On solving Eq. (12) $\widehat{w}(x, \omega)$ is obtained as

$$
\begin{aligned}
& \widehat{w}(x, \omega) \\
& =\left(\frac{P_{w h e e l} v^{3}}{E_{R} I_{R} \omega^{4}-k_{1} \omega^{2} v^{2}+k(\omega) v^{4}-\rho_{R} \omega^{2} v^{4}+\iota c_{R} \omega v^{4}}\right) e^{-i \omega\left(\frac{x}{v}\right)}
\end{aligned}
$$

Now, the rail beam deflection in time domain can be evaluated using Eq. (3) as follows

$$
w(x, t)=\frac{1}{2 \pi} \int_{-\infty}^{\infty} \widehat{w}(x, \omega) e^{\iota \omega t} d \omega
$$

Now, while evaluating the above integral $w(x, \omega)$ may encounter singularities at certain $\omega$ values. As shown by Kausel and Roësset 1992, to circumvent those singularities a complex frequency component ' $\iota \omega_{I}$ ' has been introduced in the above equation

$$
w(x, t)=\left(\frac{1}{2 \pi} \int_{-\infty}^{\infty} \widehat{w}\left(x,\left(\omega-\iota \omega_{I}\right)\right) e^{\iota \omega t} d \omega\right) e^{\omega_{I} t}
$$

where $\omega_{I}$ is a small, positive number. Finally, the above integral is evaluated by using inbuilt INTEGRAL function of MATLAB which is based on a global adaptive quadrature numerical integration scheme.

\section{Results and Discussion}

The rail-beam deflection response of the proposed model evaluated using the aboveformulation is presented in this section. The various parameters used in this study are adopted from the previous studies (Esveld 2001; Dimitrovová and Varandas 2009; Costa et al. 2015) and tabulated in Table 1. It may be mentioned here that the results are evaluated at two different values of stiffness, $k_{S_{1}}$ and $k_{S_{2}}$, which corresponds to the soft and stiff subgrade, respectively. Furthermore, to present the results in non-dimensional form, following additional parameters are considered: (a) critical velocity, $v_{c r}=$ $\left(\left(\sqrt{4 E_{R} I_{R} k_{\text {static }}}+k_{1}\right) / \rho_{R}\right)^{\frac{1}{2}}$; (b) coefficient of critical viscous damping, $c_{c r}=$ $\left(2 k_{\text {static }} \rho_{R}\right)^{\frac{1}{2}}$; (d) velocity ratio, $\alpha=v / v_{c r}$; (e) and damping ratio, $\zeta=c / c_{c r}$. Here, 
$k_{\text {static }}$ denotes the equivalent stiffness of the substructure at $\omega=0$ (see Eq. (11)), i.e., $k_{\text {static }}=k(\omega=0)=k_{B} k_{S} /\left(k_{B}+k_{S}\right)$.

Table 1. Track Parameters (Esveld 2001; Dimitrovová and Varandas 2009; Costa et al. 2015)

\begin{tabular}{|c|c|c|c|}
\hline Parameters & Values & Parameters & Values \\
\hline Axle Load, $\mathbf{2 P}_{W}$ & $2.5 \times 10^{5} \mathrm{~N}$ & $\begin{array}{l}\text { Mass of subgrade per unit } \\
\text { beam length, } \boldsymbol{\rho}_{S}\end{array}$ & $1900 \mathrm{~kg} / \mathrm{m}$ \\
\hline $\begin{array}{l}\text { Mass of rail beam per } \\
\text { unit length, } \boldsymbol{\rho}_{\boldsymbol{R}}\end{array}$ & $60 \mathrm{~kg} / \mathrm{m}$ & $\begin{array}{l}\text { Shear modulus of ballast, } \\
\boldsymbol{G}_{\boldsymbol{B}}\end{array}$ & $62.5 \mathrm{MPa}$ \\
\hline $\begin{array}{l}\text { Modulus of Elasticity of } \\
\text { rail beam, } \boldsymbol{E}_{R}\end{array}$ & $210 \mathrm{GPa}$ & Height of ballast layer, $\boldsymbol{H}_{\boldsymbol{B}}$ & $0.30 \mathrm{~m}$ \\
\hline $\begin{array}{l}\text { Moment of Inertia of rail } \\
\text { beam, } \boldsymbol{I}_{R}\end{array}$ & $3055 \mathrm{~cm}^{4}$ & $\begin{array}{l}\text { Mass of ballast per unit } \\
\text { beam length, } \boldsymbol{\rho}_{B}\end{array}$ & $510 \mathrm{~kg} / \mathrm{m}$ \\
\hline $\begin{array}{l}\text { Stiffness } \\
\text { grade), } \boldsymbol{k}_{\boldsymbol{S}_{\boldsymbol{1}}}\end{array}$ (Soft Sub- & $0.427 \mathrm{MPa}$ & Ballast Stiffness, $\boldsymbol{k}_{\boldsymbol{B}}$ & $13 \mathrm{MPa}$ \\
\hline $\begin{array}{l}\text { Stiffness (Stiff Sub- } \\
\text { grade), } \boldsymbol{k}_{\boldsymbol{S}_{\boldsymbol{2}}}\end{array}$ & $20 \mathrm{MPa}$ & Damping Ratio, $\zeta$ & 0.05 \\
\hline
\end{tabular}

Now, to highlight the effect of incorporating the inertial characteristics on sub-structural stiffness of the rail track model, the variation of frequency-dependent equivalent stiffness is studied. Figs. 3(a) and 3(b) show the variation of the ratio (real part) of $k(\omega)$ and $k_{\text {static }}$ for the soft and stiff subgrades, respectively. It may be observed that the frequency dependent equivalent stiffness varies significantly from the overall static stiffness and therefore it is essential to consider the sub-structural dynamics while analyzing the railway track system.

In Figs. 4 and 5 the rail beam deflection responses for the proposed model are compared with the conventional Winkler model comprising of an infinite beam overlying viscoelastic spring layer (Mallik et al. 2006; Basu and Rao 2013). The response of the Winkler model is evaluated by choosing mass per unit length of the rail beam $\left(\rho_{W}\right)$ and the spring stiffness $\left(k_{W}\right)$ according to the following two cases: (a) $\rho_{W}=\rho_{R}+\rho_{B}+\rho_{S}$ and $k_{W}=k_{\text {static }}$; (b) $\rho_{W}=\rho_{R}$ and $k_{W}=k_{\text {static }}$. These two cases are henceforth referred to as Winkler model I and Winkler model II respectively. Further, the deflection response of the rail beam is presented in the form of deflection amplification factor $\Omega_{D}$ which is defined as the ratio of the absolute maximum dynamic rail-beam deflection at a particular load velocity $v$ and the absolute maximum value of deflection for the static case, i.e.,

$$
\Omega_{D}(v)=|\max (w(x, t))| /\left|\max \left(w_{\text {static }}(x)\right)\right|
$$

where $w_{\text {static }}(x)$ denotes the static rail beam deflection (which is same for all the three cases, i.e., proposed model, Winkler model I, and Winkler model II)

Fig. 4 shows the variation of $\Omega_{D}$ with $v$ for the proposed model and the considered cases of Winkler model. It may be noted that the rail beam response shown in this figure is evaluated by ignoring the shear properties associated with the ballast layer (i.e., $k_{1}=0$ ) and the damping ratio $(\zeta)$ has been chosen as 0.05 . Further, parts (a) and (b) of 


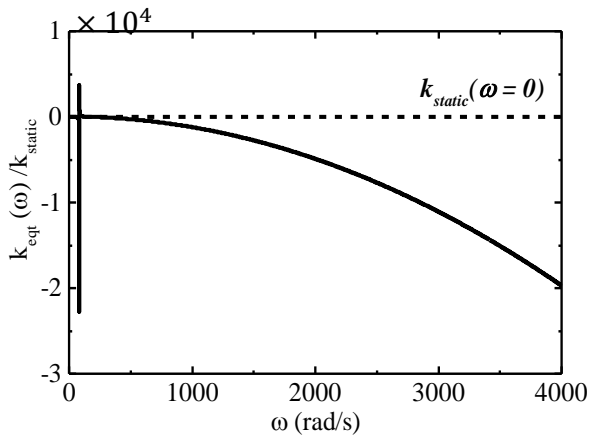

(a)

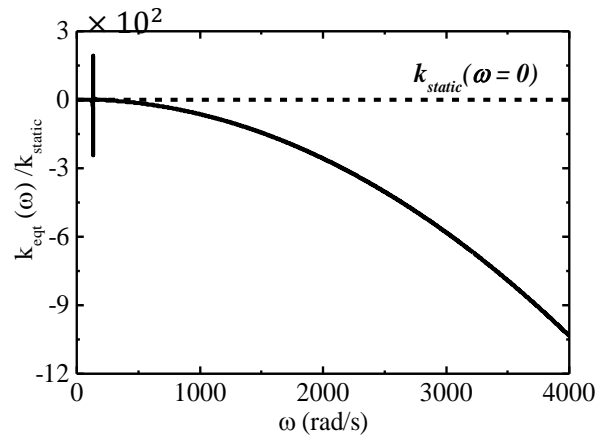

(b)

Fig. 3. Variation of frequency-dependent equivalent stiffness (real part) with loading frequency for (a) soft subgrade $\left(k_{S}=k_{S_{1}}\right)$ (b) stiff subgrade $\left(k_{S}=k_{S_{2}}\right)$

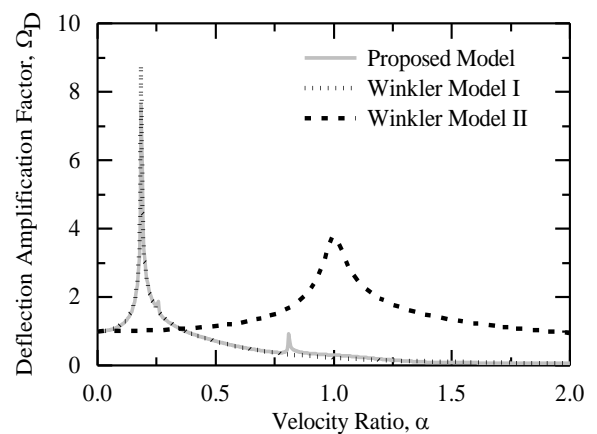

(a)

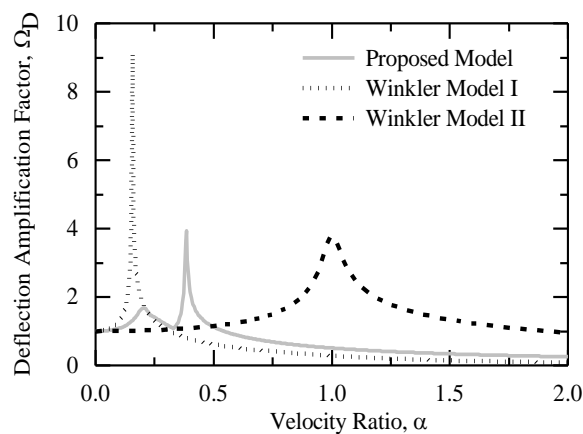

(b)

Fig. 4. Deflection amplification curves for the cases of (a) soft subgrade $\left(k_{S}=k_{S_{1}}\right)$ (b) stiff subgrade $\left(k_{S}=k_{S_{2}}\right)$

the figure show the deflection amplification curves for the cases of soft and stiff subgrades, respectively. It may be observed from Fig. 4(a) that for all the considered cases $\Omega_{D}$ increases with $\alpha$, attains a maximum value, and then decreases. The value of $\alpha$ at which the maximum amplification is attained is termed as critical velocity ratio $\left(\alpha_{c r}\right)$ of the considered model. It may be seen that in the case of Winkler model II, $\alpha_{c r}$ is equal to 1 , for which, $v=v_{c r}$. However, in the cases of proposed and Winkler model I $\alpha_{c r}$ is much smaller than 1 . Moreover, the rail beam deflection magnitudes are significantly higher (up to $140 \%$ ) especially at low velocities (say $\alpha<0.3$ ). This shows that ignoring the mass of the substructure may lead to significant underestimation in both the critical velocity values and rail beam deflections. It may be further observed that in the case of soft subgrade the deflection amplification curves for the proposed model coincides with Winkler model I. This may be explained by the fact that the stiffness value of the soft subgrade is much smaller than that of the ballast stiffness value $\left(k_{S} \ll\right.$ $k_{B}$ ). Consequently, the ballast-spring-element acts as a rigid connection between the 
ballast and subgrade mass elements (see Fig. 2(a)) and therefore the deflection amplification curve is similar to the case of Winkler model I where ballast and subgrade mass elements are lumped together with the mass of the beam.

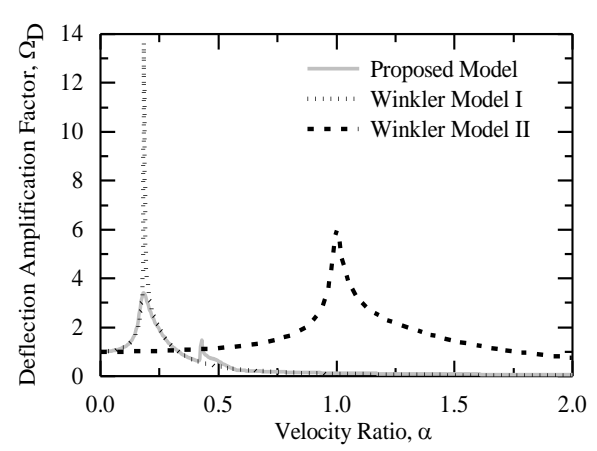

(a)



(b)

Fig. 5. Deflection amplification curves for the cases of (a) soft subgrade $\left(k_{S}=k_{S_{1}}\right)$ (b) stiff subgrade $\left(k_{S}=k_{S_{2}}\right)$

In contrast to this when the stiffness of the ballast and subgrade are of comparable magnitude the deflection amplification curve for the proposed model differ from those of the Winkler models I and II (see Fig. 4(b)). Further, on comparing Figs. 4(a) and $4(\mathrm{~b})$ it is found that the deflection magnitudes of the proposed model are much lower (by up to $600 \%$ ) in the case of stiff subgrades as compared to those in the soft subgrades. In addition to this, two peaks are observed in the deflection amplification curve (at $\alpha=0.196$ and 0.385 ) of the proposed model with the maximum deflection occurring at $0.385\left(=\alpha_{c r}\right)$. On the other hand, the respective deflection amplification curves for the Winkler models I and II do not show much variation with the subgrade stiffness.

Next, the railway track response is evaluated by incorporating the shear parameter $k_{1}$ associated with the ballast in the considered cases of railway track models (proposed model, Winkler model I, and Winkler model II). The resulting deflection amplification curves for the cases of soft and stiff subgrades are shown in Figs. 5(a) and 5(b), respectively. A comparison of Figs. 4 and 5 shows that the incorporation of the shear parameter leads to (a) change in shape of the deflection amplification curve of the proposed model, especially, when the subgrade is soft and (b) significant decrease in the deflection magnitudes (by up to $65 \%$ and $47 \%$ in the case of soft and stiff subgrades, respectively). On the other hand, the deflection amplification curves for the Winkler models do not reflect much variation both in the deflection magnitude and shape. This, therefore implies that it is essential to take into account the shear as well as inertial characteristics associated with the ballast while modelling the railway track system and the proposed model is well suited for this purpose. Finally, in the case of proposed model the critical velocities are observed at $\alpha_{c r}=0.148$ and 0.348 for soft and stiff subgrades, respectively. 


\section{Summary and Conclusions}

The effect of incorporating the inertia of the foundation on the behavior of the elastic foundation models is studied. The railway track system is idealized as an infinite EulerBernoulli beam resting on a continuous two-layer system with top and bottom layer denoting the ballast and subgrade, respectively. The ballast layer is modelled using inertial elastic shear elements and the subgrade by inertial viscoelastic elements. It is found that the incorporation of the inertial characteristics of the sub-structural system may lead to significant underestimation in the critical velocity values (by up to 85\%). Further, the deflection magnitudes and the critical velocity of the system is shown to be highly sensitive to the stiffness of the substructure. Higher deflection and lower critical velocity values are observed in the case of soft subgrade as compared to those in the stiff subgrade. Finally, the incorporation of the shear parameter associated with the ballast significantly decreases the deflection magnitudes (by up to $65 \%$ and $47 \%$ in the case of soft and stiff subgrades, respectively). However, only a slight change is seen in the critical velocity of the system.

\section{References}

1. Basu, D. and Kameswara Rao, N.S.V., 2013. Analytical solutions for Euler-Bernoulli beam on visco-elastic foundation subjected to moving load. International Journal for Numerical and Analytical Methods in Geomechanics, 37(8), pp.945-960.

2. Chen, Y.H. and Huang, Y.H., 2000. Dynamic stiffness of infinite Timoshenko beam on viscoelastic foundation in moving co-ordinate. International Journal for Numerical Methods in Engineering, 48(1), pp.1-18.

3. Chen, Y.H., Huang, Y.H. and Shih, C.T., 2001. Response of an infinite Timoshenko beam on a viscoelastic foundation to a harmonic moving load. Journal of Sound and Vibration, 241(5), pp.809-824.

4. Dimitrovová, Z. and Varandas, J.N., 2009. Critical velocity of a load moving on a beam with a sudden change of foundation stiffness: Applications to high-speed trains. Computers \& Structures, 87(19-20), pp.1224-1232.

5. Froio, D., Rizzi, E., Simões, F.M. and Da Costa, A.P., 2018. Universal analytical solution of the steady-state response of an infinite beam on a Pasternak elastic foundation under moving load. International Journal of Solids and Structures, 132, pp.245-263.

6. Kaewunruen, S. and Remennikov, A.M., 2006. Sensitivity analysis of free vibration characteristics of an in situ railway concrete sleeper to variations of rail pad parameters. Journal of Sound and Vibration, 298(1-2), pp.453-461.

7. Kargarnovin, M.H. and Younesian, D., 2004. Dynamics of Timoshenko beams on Pasternak foundation under moving load. Mechanics research communications, 31(6), pp.713-723.

8. Kenney, J.T., 1954. Steady-state vibrations of beam on elastic foundation for moving load. $J$. appl. Mech., 21, pp.359-364.

9. Mallik, A.K., Chandra, S. and Singh, A.B., 2006. Steady-state response of an elastically supported infinite beam to a moving load. Journal of Sound and Vibration, 291(3-5), pp.1148-1169.

10. Sun, L., 2001. A closed-form solution of a Bernoulli-Euler beam on a viscoelastic foundation under harmonic line loads. Journal of Sound and vibration, 242(4), pp.619-627. 
11. Timoshenko, S., 1926, September. Method of analysis of statical and dynamical stresses in rail. In Proceedings of the Second International Congress for Applied Mechanics, Zurich Switzerland (pp. 407-418).

12. Younesian, D. and Kargarnovin, M.H., 2009. Response of the beams on random Pasternak foundations subjected to harmonic moving loads. Journal of Mechanical Science and Technology, 23(11), pp.3013-3023. 